\title{
AmiD Is a Novel Peptidoglycan Amidase in Wolbachia Endosymbionts of Drosophila melanogaster
}

\begin{abstract}
Miriam Wilmes ${ }^{1 \dagger}$, Kirstin Meier ${ }^{1}{ }^{\dagger}$, Andrea Schiefer ${ }^{1}$, Michaele Josten ${ }^{1}$, Christian F. Otten ${ }^{2}$, Anna Klöckner ${ }^{3}$, Beate Henrichfreise ${ }^{3}$, Waldemar Vollmer ${ }^{2}$, Achim Hoerauf ${ }^{1,4 *}$ and Kenneth Pfarr ${ }^{1 *}$

${ }^{1}$ Institute of Medical Microbiology, Immunology and Parasitology, University Hospital Bonn, Bonn, Germany, ${ }^{2}$ Institute for Cell and Molecular Bioscience, Newcastle University, Newcastle upon Tyne, United Kingdom, ${ }^{3}$ Institute for Pharmaceutical Microbiology, University of Bonn, Bonn, Germany, ${ }^{4}$ German Center for Infection Research (DZIF), Partner Site Bonn-Cologne, Bonn, Germany
\end{abstract}

Wolbachia endobacteria are obligate intracellular bacteria with a highly reduced genome infecting many arthropod and filarial species, in which they manipulate arthropod reproduction to increase their transmission and are essential for nematode development and survival. The Wolbachia genome encodes all enzymes required for the synthesis of the cell wall building block lipid II, although a peptidoglycan-like structure has not been detected. Despite the ability to synthesize lipid II, Wolbachia from arthropods and nematodes have only a subset of genes encoding enzymes involved in the periplasmic processing of lipid II and peptidoglycan recycling, with arthropods having two more than nematodes. We functionally analyzed the activity of the putative cell wall hydrolase AmiD from the Wolbachia endosymbiont of Drosophila melanogaster, an enzyme not encoded by the nematode endobacteria. Wolbachia AmiD has $\mathrm{Zn}^{2+}$-dependent amidase activity and cleaves intact peptidoglycan, monomeric lipid II and anhydromuropeptides, substrates that are generated during bacterial growth. AmiD may have been maintained in arthropod Wolbachia to avoid host immune recognition by degrading cell wall fragments in the periplasm. This is the first description of a wolbachial lipid II processing enzyme putatively expressed in the periplasm.

Keywords: metalloenzyme, amidase, peptidoglycan, lipid II, Wolbachia, host recognition

\section{INTRODUCTION}

The genus Wolbachia represents a group of obligate intracellular Gram-negative bacteria that are widespread in arthropods and filarial nematodes. Some species of the latter infect humans and cause lymphatic filariasis (lymphedema; hydrocele) or onchocerciasis (river blindness) (Specht et al., 2013). Wolbachia endobacteria reside in host-derived vacuoles and are vertically transmitted from females to their offspring. The interaction of Wolbachia with their hosts ranges from mutualistic symbiosis to parasitism (Werren et al., 2008). In filaria, these endobacteria are required for worm development, fertility and survival, and thus filariasis can be effectively treated with antibiotics targeting Wolbachia (Taylor and Hoerauf, 2001; Taylor et al., 2010). Wolbachia in arthropods are 
largely parasitic and manipulate host reproduction by several mechanisms including feminization of genetic males or cytoplasmic incompatibility to increase the number of infected females in the population (Werren et al., 2008). However, there are exceptions and in some cases the arthropod host requires Wolbachia for oogenesis and positive benefits to fitness have also been demonstrated in terms of resistance to different pathogens and in nutrient provisioning (Zug and Hammerstein, 2015). For example, in the bedbug Cimex lectularis, Wolbachia were shown to be essential for growth and reproduction of the host by providing $\mathrm{B}$ vitamins, indicating a mutualistic relationship (Nikoh et al., 2014).

A characteristic feature of intracellular bacteria is a highly reduced genome due to adaption to the host (Stepkowski and Legocki, 2001). The Wolbachia genomes (ranging from 0.9 to $1.5 \mathrm{Mb}$ ) have lost many genes, particularly those involved in biosynthetic pathways (Wu et al., 2004; Foster et al., 2005; Lindsey et al., 2016). However, the genomes of these endobacteria encode all enzymes required for the synthesis of the cell wall precursor lipid II although a functional cell wall has not been detected. Intracellular bacteria do not need a cell wall for osmotic stabilization, but it has been shown that lipid II is crucial in Wolbachia for coordinated cell division (Vollmer et al., 2013).

The major component of bacterial cell walls is peptidoglycan (PG), a polymer consisting of glycan strands with alternating units of $\mathrm{N}$-acetylglucosamine (GlcNAc) and $\mathrm{N}$-acetylmuramic acid (MurNAc) that are connected via peptides attached by an amide linkage to the lactyl group of MurNAc. The peptides of Gram-negative bacteria typically contain L-alanine, D-glutamate, meso-diaminopimelic acid (mDAP) and D-alanine (Typas et al., 2012).

PG biosynthesis is a multi-step process involving reactions in the cytoplasm and both sides of the cytoplasmic membrane. It starts in the cytoplasm with assembly of the soluble cell wall precursors UDP-GlcNAc and UDP-MurNAc-pentapeptide. These precursors and the membrane carrier undecaprenyl phosphate are utilized to produce lipid II [undecaprenylpyrophosphoryl-MurNAc-(pentapeptide)-GlcNAc] at the inner leaflet of the cytoplasmic membrane. Lipid II is then translocated across the membrane and incorporated into the growing peptidoglycan network by glycosyltransferase and transpeptidation reactions (Typas et al., 2012).

Cell wall hydrolases such as lytic transglycosylases, endopeptidases and amidases are capable of cleaving different bonds within the net-like peptidoglycan structure. They allow for the separation of daughter cells at the end of cell division and release turnover products during cell growth, which are subsequently translocated into the cytoplasm via permeases and recovered by several recycling enzymes (Vollmer et al., 2008; Johnson et al., 2013). Lytic transglycosylases cleave the glycosidic bond between MurNAc and GlcNAc units, amidases and endopeptidases hydrolyze various amide bonds in the PG (Vollmer et al., 2008).

Escherichia coli produces five N-acetylmuramoyl-L-alanine amidases. The periplasmic amidases AmiA, AmiB and AmiC are involved in septum cleavage during cell division and can substitute for each other in function (Heidrich et al.,
2001; Priyadarshini et al., 2007). The cytoplasmic AmpD participates in the recycling of PG fragments and uses only anhydromuropeptides as a substrate, which are characterized by a glycosidic bond between $\mathrm{C} 1$ and $\mathrm{C} 6$ of MurNAc as the result of glycan chain cleavage by lytic transglycosylases (Jacobs et al., 1995). The lipoprotein AmiD is anchored to the outer membrane and has a broad substrate spectrum, but its precise role is unknown (Uehara and Park, 2007). Other Gram-negative bacteria encode only a single periplasmic $\mathrm{N}$-acetylmuramoyl-Lalanine amidase. In Neisseria gonorrhoeae AmiC functions in cell separation and PG fragment release (Lenz et al., 2016), while in Vibrio cholerae AmiB is crucial for cell division and growth (Möll et al., 2014). In intracellular pathogens of the genus Chlamydia, AmiA has, in contrast to free-living bacteria, a dual enzymatic activity acting as an $\mathrm{N}$-acetylmuramoyl-L-alanine amidase and a DD-carboxypeptidase (Klöckner et al., 2014).

The genome of Wolbachia from Drosophila melanogaster $(w \mathrm{Mel})$ has retained only one predicted periplasmic cell wall hydrolase (WD1073). Sequence alignments with periplasmic E. coli AmiA, AmiB, AmiC and AmiD reveal the highest homology to E. coli AmiD (27\% sequence identity). Sequenced genomes of filarial Wolbachia residing in Brugia malayi $(w \mathrm{Bm})$ and Onchocerca volvulus ( $w \mathrm{Ov}$ ) show that these strains have lost the ability to synthesize any of these enzymes, e.g., $w \mathrm{Bm} 0682$ might encode an amidase in $w \mathrm{Bm}$, but genome analysis has concluded that it is a pseudogene (Figure 1; Wu et al., 2004; Foster et al., 2005).

In this study, we analyzed the activity of AmiD from $w \mathrm{Mel}$. Our results demonstrate that recombinantly expressed AmiD from Wolbachia $\left(\mathrm{AmiD}^{\mathrm{wol}}\right.$ ) cleaves the MurNAc-L-Ala bond in PG, monomeric lipid II and anhydromuropeptides. The amidase activity is $\mathrm{Zn}^{2+}$-dependent and inhibited in the presence of the metal chelators EDTA and 1,10-phenanthroline. The enzymatic activity of $\mathrm{AmiD}^{\mathrm{wol}}$ may have a crucial role in cleavage of a PG-like structure and allow Wolbachia to avoid host organism immune responses by degrading cell wall fragments in the periplasm that could be recognized by innate immune receptors (Buchon et al., 2014).

\section{MATERIALS AND METHODS}

\section{In silico Analysis of AmiD ${ }^{\text {wol }}$ Localization}

We used BLAST analysis (http://blast.ncbi.nlm.nih.gov/Blast.cgi) to identify sequences similar to WD1073 and the Clustal Omega multiple sequence alignment tool (http://www.ebi.ac.uk/Tools/ $\mathrm{msa} /$ clustalo/) to align $\mathrm{AmiD}$ sequences of different species. A signal peptide for periplasmic secretion and cleavage site was predicted by SignalP (http://www.cbs.dtu.dk/services/SignalP-3. $0 /)$.

\section{Cloning and Expression of AmiDwol}

The gene amiD from $w \mathrm{Mel}$ was amplified without its native signal sequence using the primers listed in Table 1. The PCR product was cloned into the expression vector pASK-IBA2C (IBA Lifesciences, Göttingen, Germany) using the BsaI restriction sites in the primers to generate a protein with an $\mathrm{N}$-terminal OmpA 


\section{Signal peptide}

\begin{tabular}{|c|c|c|}
\hline wMel & - & 32 \\
\hline wRi & MILSCKE IQQPYSNYIMKNYLQSVYEDGMKIQLSKVNKYLLVLLITVSAFLISGQVSSSS & 60 \\
\hline wPip & $--1,-M K L I V K K C L S--$ VLFLLLLVAPL LYGQQSCLS & 30 \\
\hline $\mathrm{wBm}$ & 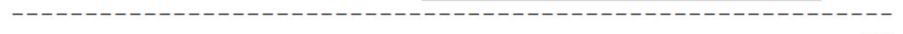 & 0 \\
\hline R.helvetica & 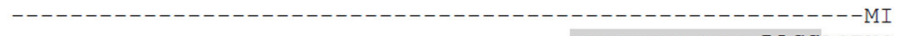 & 2 \\
\hline E.coli & $\begin{array}{c}\text { H79 } \\
\text { HAGCAGEKG }\end{array}$ & 22 \\
\hline wMel & NIENDFQDLQEKLPLLKDQDLLFLDPASALNYGDRAGKKVLMVIVH堋TETSTLKGTKDTL & 92 \\
\hline wRi & NIENDFQDLQEKLPLLKDQDLLFLDPASALNYGDRAGKKVLMVIVH TETSTLKGTKDTL & 120 \\
\hline wPip & DIENDFQDLKTSL-----KNPIFLDPAPSLNYDDREGKKVLMVVVH HTESPTLKSTKYAL & 85 \\
\hline $\mathrm{wBm}$ & 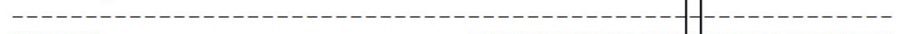 & 0 \\
\hline R.helvetica & 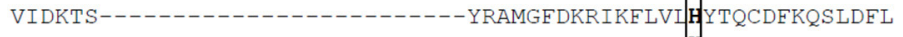 & 37 \\
\hline E.coli & 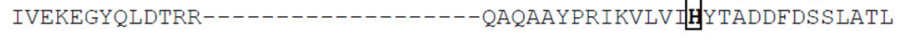 & 63 \\
\hline wMel & NARGLSVHF IVD-------RDGS I TLMVPLEKEAWHAG ISYARVKVDSKLEELRKLNNYS & 145 \\
\hline wRi & NARGLSVHFIVD------RDGNI TLMVPLEKEAWHAGISYARVKVDSKLEELRKLNNYS & 173 \\
\hline wPip & NSSGISVQLIVD-------RDGS I TLMVPLEKRAWHAGISYAKVQVDNVVEELRKLNDYS & 138 \\
\hline $\mathrm{wBm}$ & 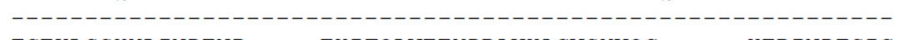 & \\
\hline R.helvetica & TGEKLSSHYLINDENP-----EHIFQLVEEYDRAWHAGVSYWQG------HERINDTSIG & 84 \\
\hline E.coli & TDKQVSSHYLVPAVPPRYNGKPRIWQLVPEQELAWHAGISAWR--------GATRLNDTS & 115 \\
\hline & $\begin{array}{lll}\text { H187 } & \text { H18 }\end{array}$ & \\
\hline wMel & VGIEIVNTGL--------EPFPEEQMRSVKELILYLMERFKIKRDMIFSAFSEIGTIVYD & 196 \\
\hline wRi & VGIEIVNTGL--- -- - EPFPEEQMRSVKEL I LYLMERFKIKRDMIFSHSEIGTIVYD & 224 \\
\hline wPip & IGIEIVNTGL--------EPFPEEQMKSVKDLILYLMKRFKIRKDMIFSH $A$ EIGTIVYN & 189 \\
\hline $\mathrm{wBm}$ & 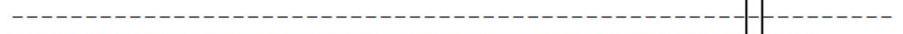 & 0 \\
\hline R.helvetica & IEIVNPAFEVNAKNNDIIWLPYSKPOINSVISLCKOIIARYDIKPTRVVAHSDIA----- & 141 \\
\hline E. coli & IGIELENRGWQKSAGVKYFAPFE PAQIQALIPLAKDI I ARYH IKPENVVAHADIA----- & 170 \\
\hline wMel & $\begin{array}{c}\text { D207 } \\
\text { PE-LGYTMRK FDPHKLFDWELLEKNE I GLH I SDRINPKDAKHKMGKTLYKAGDRNEG--- }\end{array}$ & 252 \\
\hline WRi & PE-LGYTMRKADPHKLFDWELLEKNEIGLHISDRINPKDAKHKMGKTLYKAGDRNEG--- & 280 \\
\hline wPip & SAIEGYAMRKAD|PHKLFDWELLEKSGIGLHIGDRISPQDAEQKVNEVLYKVGDKSEN--- & 246 \\
\hline wBm & 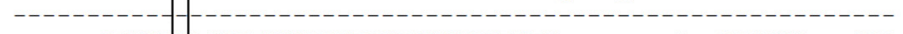 & \\
\hline R.helvetica & ----- PGRKdD|PGPLFPWKLLYDNG IGAWYDYRSFN--------D--LLSQVD---ITD & 180 \\
\hline E. coli & -----PQRKLDPGPLEPWQQLAQQGIGAWPDAQRV---------NFYLAGRAPHTPVD & 214 \\
\hline wMel & ILKLKQRLNRFFYKIEPWNDKRGNVIF PDNNADYS DEFDENFVWVIYQFS I HNLPREIRK & 312 \\
\hline wRi & ILKLKQRLNRFFYKIEPWNDKKGNVIF PDNNADYSDEFDENFVWVIYQFS IHNLPREIRK & 340 \\
\hline wPip & ILKLKKRLNGFFYKILPWNDREGNMIF PDNNANYSNEFDENFAWVINQFSMHHLPKEIRK & 306 \\
\hline wBm & & 0 \\
\hline R.helvetica & IKAVQQKFITYGYKLEATG---------------ILDSKMKDVI ISFQMHFRPSNFSG & 223 \\
\hline E.coli & TASLIELLARYGYDVKPDMTPR------- & 256 \\
\hline wMel & DLPLKLEQADIFPEFFSEYSHGISSSYLTFSEKIKSTLQ-PCLSKVDYENLLSSLAQYEN & 371 \\
\hline wRi & DLPLKLEQADIFPEFFSEYSHGISSSYLTESEKIKSTLQ-PCLSKVDYENLLSSLAQYEN & 399 \\
\hline WRip & DLPLKLEQEDILPEFLVKYRNLIFSEFLSLSDKTQLSLKP FELNEEDYKHLLSSLAEYEN & 366 \\
\hline R.helvetica & DLD--IETIAILDALILKYKSELSS--- & 248 \\
\hline E.coli & 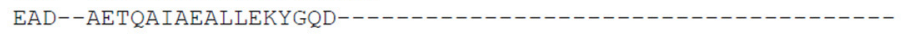 & 276 \\
\hline wMel & NISPDASTTLMYKIKLYYGSYLRYRIWSSLYKPFKLNVLEELEILKSGVLSLKSLDSSKA & 431 \\
\hline wRi & NISPDASTTLMYKIKLYYDSYLRYRIWSSLYKPFKLNVLEELEILKSGVLSLKSLDSSKA & 459 \\
\hline wPip & NISSDAFAMLMYKIKLYYDSYLRYNIRSSLYIPFRLNSFEKLDILKNEILSFKSISPEKA & 426 \\
\hline wBm & --------MYNIKLYYSSYLRYNIRSSLYIPFKPNI LVKLDILKNEVLSLKTVSPEKV & 50 \\
\hline R.helvetica & 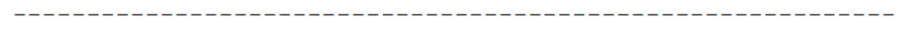 & 248 \\
\hline E.coli & & 276 \\
\hline WMel & AEVSSLIDSFKVDIS LEFQGFEKQWFQE FKNAWRQEF I PS LEEQITWTALHEA I LEYLEK & 491 \\
\hline wRi & AEVSSLIDSFKVDISLEFQGFEKQWFQEFKNAWRQEF I PS LEEQI TWTALHEA I LEYLEK & 519 \\
\hline wPip & IEVSNLINEFRSKI LSDFQNFEKQWWEEEKDTWKQKKF I PNMERQMSWTALHETVLEYLEK & 486 \\
\hline wBm & VEVSNLIDKFRLKILTDFQNFEKQWFQEFKNTWKQKLMPNMRSEYL--GLHCTKIY---- & 104 \\
\hline R.helvetica & - & 248 \\
\hline E. COIi & --------------- & 276 \\
\hline wMel & AKEEIR & 497 \\
\hline wRi & AKEEIR & 525 \\
\hline wPip & AKKEV- & 491 \\
\hline $\mathrm{wBm}$ & ------ & 104 \\
\hline R.helvet & ----- & 248 \\
\hline E.coli & ---- & 276 \\
\hline
\end{tabular}

FIGURE 1 | Amino acid sequence alignment of AmiD from E. coli and selected Wolbachia species. Four Wolbachia AmiD sequences (wMel, Wolbachia from Drosophila melanogaster, WD1073; wRi, Wolbachia from Drosophila simulans, EAL60110; wPiP, Wolbachia from Culex quinquefasciatus Pel, CAQ54941; wBm, Wolbachia from Brugia malayi, AAW71270) and one Rickettsia species (R. helvetica, WP_010420485.1) were aligned with that of E. coli (NP_415388).The signal peptides of Wolbachia AmiD (shaded in gray) were predicted by SignalP (http://www.cbs. dtu.dk/services/SignalP/). The E. coli AmiD cysteine-containing lipobox motif at the $\mathrm{C}$-terminal end of the signal sequence is underlined. The amidase active site of $E$. coli AmiD contains three zinc-coordinating residues (H50, H166, D176). These active site residues (boxed) are conserved among insect Wolbachia (in wMel: H79, H187, D207). 
TABLE 1 | Primers used to clone amiD into expression vectors and for mutagenesis of active site residues.

\begin{tabular}{ll}
\hline Primer name & Sequence $\left(\mathbf{5}^{\prime} \rightarrow \mathbf{3}^{\prime}\right)$ \\
\hline IBA2C_amiD- & ATGGTAGGTCTCAGGCCTCAAGCAATATCGAGAATGATTTCA \\
for_2 & \\
IBA2C_amiD- & ATGGTAGGTCTCAGCGCTACGAATTCTTCCTTGCTTTTCTA \\
rev_2 & \\
\hline amiD_mut3-for & GGTTATAGTTCACGCGACTGAAACATCAAC \\
$($ H79A) & \\
amiD_mut3-rev & GTTGATGTTCAGTCGCGTGAACTATAACC \\
$($ H79A)
\end{tabular}

amiD_mut5-for GCTATACAATGCGTAAACCAGCGCCACACAAATTGTTTGATTG (D207A)

amiD_mut5-rev CAATCAAACAATTTGTGTGGCGCTGGTTACGCATTGTATAGC (D207A)

IBA3_amiD-for ATGGTAGGTCTCAAATGATGAAAATCCAACTATCTAAAGTCAAC IBA3_amiD-rev ATGGTAGGTCTCAGCGCTACGAATTCTTCCTTGCTIITCTA

Bsal restriction sites are written in bold. Mutated positions are underlined.

leader peptide for periplasmic secretion and a C-terminal Streptag for purification. To test whether $\mathrm{AmiD}^{\text {wol }}$ is secreted into the periplasm, amiD from $w \mathrm{Mel}$ was cloned with its native signal sequence into the cytoplasmic expression vector pASK-IBA3plus (IBA Lifesciences) using the BsaI restriction sites.

\section{Site-Directed Mutagenesis}

$\mathrm{H} 79$ and D207 in $\mathrm{AmiD}^{\text {wol }}$ were changed to alanine using the QuikChange Lightning Site-Directed Mutagenesis Kit (Agilent Technologies, Waldbronn, Germany) according to the manufacturer's instructions. Primers are listed in Table 1. Correct base changes were confirmed by sequencing (SeqLab, Göttingen, Germany).

\section{Co-Solvent Assisted Overproduction and Purification of AmiDwol}

E. coli JM83 (DSM3947) containing pASK-IBA2C_amiD or pASK-IBA2C (empty vector) was grown in $2 \mathrm{~L}$ salt-free LB medium containing $342 \mathrm{mM}$ D-mannitol (equivalent to $342 \mathrm{osm} / \mathrm{L} / 10 \mathrm{~g} / \mathrm{L}$ sodium chloride) and $30 \mu \mathrm{g} / \mathrm{mL}$ chloramphenicol at $25^{\circ} \mathrm{C}$ (Otten et al., 2015). Expression of $\mathrm{AmiD}^{\text {wol }}$ was induced at $\mathrm{OD}_{600} 0.6$ by addition of $200 \mathrm{ng} / \mathrm{mL}$ anhydrotetracycline (AHT; IBA Lifesciences, Göttingen, Germany) and cells were incubated at $25^{\circ} \mathrm{C}$ until they started lysing due to in vivo activity of $\mathrm{AmiD}^{\text {wol }}$.

Purification of Strep-tagged proteins was performed according to the manufacturer's instructions for periplasmic expression of metalloproteins (IBA Lifesciences). Cell pellets were suspended in $10 \mathrm{~mL}$ buffer $\mathrm{P}(100 \mathrm{mM}$ Tris- $\mathrm{HCl}, \mathrm{pH}$ $8 ; 500 \mathrm{mM}$ sucrose; $2 \mathrm{mg} / \mathrm{mL}$ polymyxin B sulfate; $1 \mathrm{mg} / \mathrm{mL}$ lysozyme) and incubated for $30 \mathrm{~min}$ on ice. To reduce viscosity, benzonase $(20 \mathrm{U} / \mathrm{mL})$ was added and the suspension was incubated for $15 \mathrm{~min}$ on ice. Cleared lysate containing the
Strep-tagged proteins was prepared by centrifugation $(38,800 \mathrm{~g}$, $\left.15 \mathrm{~min}, 4^{\circ} \mathrm{C}\right)$.

Proteins were purified by gravity flow chromatography at $4^{\circ}$ C. One mL Strep-Tactin ${ }^{\circledR}$ Sepharose (IBA Lifesciences) was applied to a $1 \mathrm{~mL}$ polypropylene column and equilibrated with $2 \mathrm{~mL}$ buffer W (100 mM Tris- $\mathrm{HCl}, \mathrm{pH} 8 ; 150 \mathrm{mM} \mathrm{NaCl}$ ). The cleared lysate was loaded onto the column and washed five times with $1 \mathrm{~mL}$ buffer W. Finally, AmiD ${ }^{\text {wol }}$ was eluted six times with $0.5 \mathrm{~mL}$ buffer E (100 mM Tris-HCl, $\mathrm{pH} 8 ; 150 \mathrm{mM} \mathrm{NaCl}, 2.5 \mathrm{mM}$ desthiobiotin). AmiD ${ }^{\text {wol }}$ was stored in $50 \%$ glycerol at $-20^{\circ} \mathrm{C}$ and was stable for at least 6 months.

\section{Growth Kinetics}

E. coli JM83 harboring periplasmic expression vectors was grown in LB medium supplemented with $30 \mu \mathrm{g} / \mathrm{mL}$ chloramphenicol at $25^{\circ} \mathrm{C}$ and the $\mathrm{OD}_{600}$ was measured every $30 \mathrm{~min}$. AmiD wol expression was induced with $200 \mathrm{ng} / \mathrm{mL}$ AHT at an $\mathrm{OD}_{600}$ of 0.6.

\section{Lipid II Synthesis}

Lipid II was synthesized in vitro as described previously (Schneider et al., 2004). Briefly, isolated membranes from Micrococcus flavus (Schneider et al., 2004) were incubated with UDP-MurNAc-pentapeptide from Staphylococcus simulans 22 or Bacillus cereus T (Schneider et al., 2004), $\mathrm{C}_{55}-\mathrm{P}$ (Larodan, Solna, Sweden) and UDP-GlcNAc (Sigma-Aldrich, Taufkirchen, Germany). Optimal conditions had to be titrated in an analytical assay before preparing lipid II in a larger scale. Lipid II was obtained by mixing 20-30 $\mu \mathrm{l}$ isolated membranes, $7.5-15 \mu \mathrm{l}$ UDP-MurNAc-pentapeptide, $5 \mathrm{nmol} \mathrm{C}_{55}$-P, $1 \mathrm{mM}$ UDP-GlcNAc, $5 \mathrm{mM} \mathrm{MgCl}_{2}, 60 \mathrm{mM}$ Tris (pH 7.5), 0.5\% Triton X-100 (v/v) in a final volume of $75 \mu \mathrm{l}$. The mixture was incubated for $4 \mathrm{~h}$ at $30^{\circ} \mathrm{C}$ with shaking and afterwards extracted and visualized by thin layer chromatography (TLC) as described previously (Schneider et al., 2004; Klöckner et al., 2014). Lipid II synthesis in a preparative scale was achieved by using a 200 -fold volume of the analytical scale.

Purification of lipid II was performed on a $5 \mathrm{~mL}$ HiTrap DEAE FF column (GE Healthcare, Freiburg, Germany) and eluted with a linear gradient of chloroform/methanol/water $(2: 3: 1, \mathrm{v} / \mathrm{v})$ to chloroform/methanol/300 $\mathrm{mM}$ ammonium bicarbonate (2:3:1, $\mathrm{v} / \mathrm{v})$. Lipid II was quantified by measuring the released phosphate upon total hydrolysis (Rouser et al., 1970).

\section{AmiD ${ }^{\text {wol }}$ Activity Against PG or Lipid II}

Preparation and staining of sacculi with Remazol Brilliant Blue (RBB) using E. coli W3110 (DSM3947) was performed as described previously (Uehara et al., 2009, 2010). For the dyerelease assays, $20 \mu \mathrm{L}$ of stained PG sacculi were incubated at $30^{\circ} \mathrm{C}$ overnight with $4 \mu \mathrm{M}$ of purified $\mathrm{AmiD}^{\text {wol }}$ in a final volume of $200 \mu \mathrm{L}$ containing $50 \mathrm{mM}$ Tris (pH 7.5) and 5\% dimethyl sulfoxide (DMSO). Samples were centrifuged [20,000 g, $20 \mathrm{~min}$, room temperature (RT)] and absorbance of the supernatants was measured at $595 \mathrm{~nm}$.

Using lipid II as a substrate for activity assays, $4 \mu \mathrm{g}$ purified $\mathrm{AmiD}^{\text {wol }}, 2 \mathrm{nmol}$ lipid II, $50 \mathrm{mM}$ Tris (pH 7.5) and 5\% DMSO in a volume of $40 \mu \mathrm{L}$ were incubated for $4 \mathrm{~h}$ at $30^{\circ} \mathrm{C}$. Reaction products were extracted with $40 \mu \mathrm{L}$ of n-butanol/pyridine acetate 
$(2: 1 \mathrm{v} / \mathrm{v}, \mathrm{pH} 4.2)$, centrifuged (21,000 g, $5 \mathrm{~min}, \mathrm{RT})$ and analyzed by TLC and mass spectrometry (MS) as described previously (Klöckner et al., 2014).

To inhibit amidase activity of $\mathrm{AmiD}^{\text {wol }}, 1 \mathrm{mM}$ EDTA was added to buffer $\mathrm{P}$, buffer $\mathrm{W}$ and buffer $\mathrm{E}$ during lysis and purification of the protein according to the manufacturer's instructions (IBA Lifesciences). $5 \mathrm{mM}$ 1,10-phenanthroline (Sigma-Aldrich) was added to the reaction mixtures of the activity tests described above.

\section{Cleavage of Anhydromuropeptides}

PG sacculi from E. coli were prepared as described (Glauner, 1988). The PG $(750 \mu \mathrm{g})$ was digested with the lytic transglycosylase Slt $(1 \mu \mathrm{M})$ in a final volume of $210 \mu \mathrm{L}$ containing $10 \mathrm{mM}$ HEPES ( $\mathrm{pH} 7.5$ ) and $150 \mathrm{mM} \mathrm{NaCl}$ for $18 \mathrm{~h}$ at $37^{\circ} \mathrm{C}$. The reaction mixture was heated for $10 \mathrm{~min}$ at $100^{\circ} \mathrm{C}$ and centrifuged at $17,000 \mathrm{~g}$ for $20 \mathrm{~min}$. The supernatant containing the 1,6-anhydro-muropeptides was collected and stored at $2-8^{\circ} \mathrm{C}$.

1,6-Anhydro-muropeptides $(15 \mu \mathrm{L})$ were incubated with $\mathrm{AmiD}^{\text {wol }}(2 \mu \mathrm{M})$ in a final volume of $50 \mu \mathrm{L}$ containing $50 \mathrm{mM}$ Tris $\left(\mathrm{pH} \mathrm{7.5)}\right.$ and $5 \%$ DMSO for $4 \mathrm{~h}$ at $30^{\circ} \mathrm{C}$. Samples were boiled for $10 \mathrm{~min}$, centrifuged for $20 \mathrm{~min}$ and the supernatant recovered. The $\mathrm{pH}$ of the supernatant was adjusted to $\mathrm{pH} 4$ with $20 \%$ phosphoric acid. HPLC analysis was carried out as described (Glauner, 1988), and selected peaks were collected and analyzed by mass spectrometry (Bui et al., 2009).

\section{Mass Spectrometry}

For detection of reaction products from $\mathrm{AmiD}^{\text {wol }}$ activity assays using lipid II, $1 \mu \mathrm{L}$ of the sample was placed onto a ground steel matrix assisted laser desorption ionization time-of-flight target plate (Bruker Daltonik, Bremen, Germany) and allowed to dry at RT. Each sample was then overlaid with $1 \mu \mathrm{L}$ of matrix (saturated solution of 6-Aza-2-thiothymine in 50\% ethanol/20 mM diammonium citrate or alpha-cyano-cinnamic acid in $33 \%$ acetonitrile $/ 0.1 \%$ trifluoroacetic acid) and air dried at RT. Spectra were recorded in the reflector negative mode on a Biflex III mass spectrometer (Bruker Daltonik).

\section{Statistical Analysis}

For statistical analysis, GraphPad Prism 5 software (GraphPad Software Inc., La Jolla, CA, USA) was used. Statistical differences in bacterial growth and enzymatic activities were determined using the Student's $t$-test with $P<0.05$.

\section{RESULTS}

$\mathrm{AmiD}^{\mathrm{wol}}$ is a predicted 497 amino acid protein with $27 \%$ amino acid sequence identity with the E. coli AmiD sequence and contains a predicted $\mathrm{N}$-terminal signal sequence at amino acids 1-30 for secretion into the periplasm (Figure 1). However, the $\mathrm{AmiD}^{\text {wol }}$ signal sequence lacks a lipobox motif with a conserved cysteine residue for lipidation and subsequent insertion into the outer membrane (E. coli AmiD "L ${ }^{14} \mathrm{AGC}^{17 " ; ~ F i g u r e ~ 1) ~}$ suggesting that $\mathrm{AmiD}^{\mathrm{wol}}$ is not a lipoprotein.

\section{AmiD ${ }^{\text {wol }}$ Is Secreted into the E. coli Periplasm}

To test if $\mathrm{AmiD}^{\mathrm{wol}}$ is transported into the periplasm after expression, the protein was overproduced with its native $\mathrm{N}$ terminal signal sequence in E. coli JM83 using the expression vector pASK-IBA3plus. The cells were harvested and the outer membrane and cell wall were disrupted by the antibiotic polymyxin B and lysozyme, respectively, to release the periplasmic proteins. Western Blot analysis confirmed the presence of the soluble $\mathrm{AmiD}^{\text {wol }}$ in the periplasmic fraction (Supplementary Figure 1).

\section{AmiD wol Is Functional In vitro and In vivo}

For functional analysis of $\mathrm{AmiD}^{\text {wol }}$, the enzyme was produced in $E$. coli by co-solvent assisted periplasmic expression as previously established for chlamydial proteins (Otten et al., 2015). For this, the native signal sequence of $a m i D$ was replaced with the ompA signal sequence of the expression vector pASK-IBA2C and mannitol was used as co-solvent for protein stabilization.

When $\mathrm{AmiD}^{\text {wol }}$ was overexpressed by addition of AHT, the turbidity of the E. coli culture decreased gradually, indicating $\mathrm{AmiD}^{\text {wol }}$ hydrolytic activity on PG and subsequent lysis of the host cells. In contrast, cells carrying the empty vector pASKIBA2C or the plasmid for the expression of an inactive version of $\mathrm{AmiD}\left(\mathrm{AmiD}^{\mathrm{wol}}\right.$-H79A or AmiD ${ }^{\text {wol }}$-D207A in which one of the zinc-coordinating residues was replaced by alanine) did not show any growth defects (Figure 2).

$\mathrm{AmiD}^{\text {wol }}$ was purified by Strep-tag affinity chromatography. The activity of purified AmiD wol was analyzed in a dye-release assay by incubating the enzyme with RBB-stained PG from $E$. coli. Released reaction products in the supernatant resulting from PG cleavage were quantified by absorbance measurement (Figure 3A). Recombinant $\mathrm{AmiD}^{\text {wol }}$ was fully active at $\mathrm{pH} 7$ 9 and temperatures ranging from 20 to $37^{\circ} \mathrm{C}$ (Supplementary

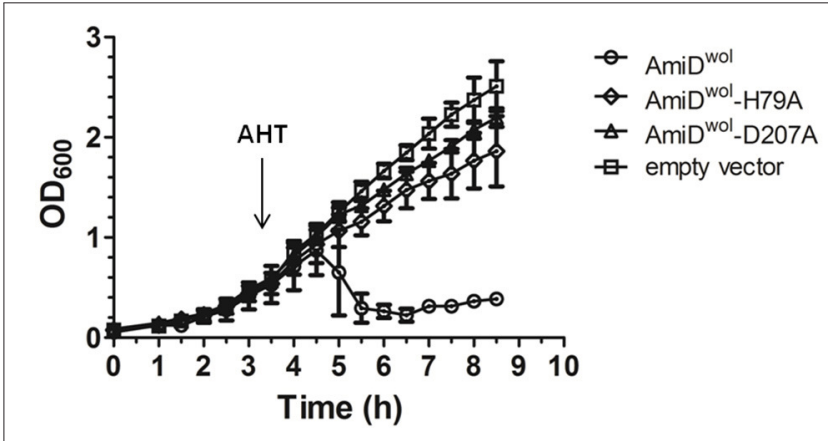

FIGURE 2 | AmiD ${ }^{\text {wol }}$ expressed in E. coli has in vivo activity. Growth kinetics of $E$. coli JM83 containing expression vectors for periplasmic targeting of proteins. Protein expression was induced at an $\mathrm{OD}_{600}$ of 0.6 with anhydrotetracycline (AHT). Periplasmic overexpression of AmiDwol resulted in lysis of host cells compared to the amidase active-site mutants AmiD ${ }^{\text {Wol }}$ H79A and $\mathrm{AmiD}^{\text {Wol }}$-D207A and the empty vector control. $\mathrm{OD}_{600}$ was measured every $30 \mathrm{~min}$ for $8.5 \mathrm{~h}$. Each point represents mean $\pm \mathrm{SD}$. The graph is representative of three experiments. 

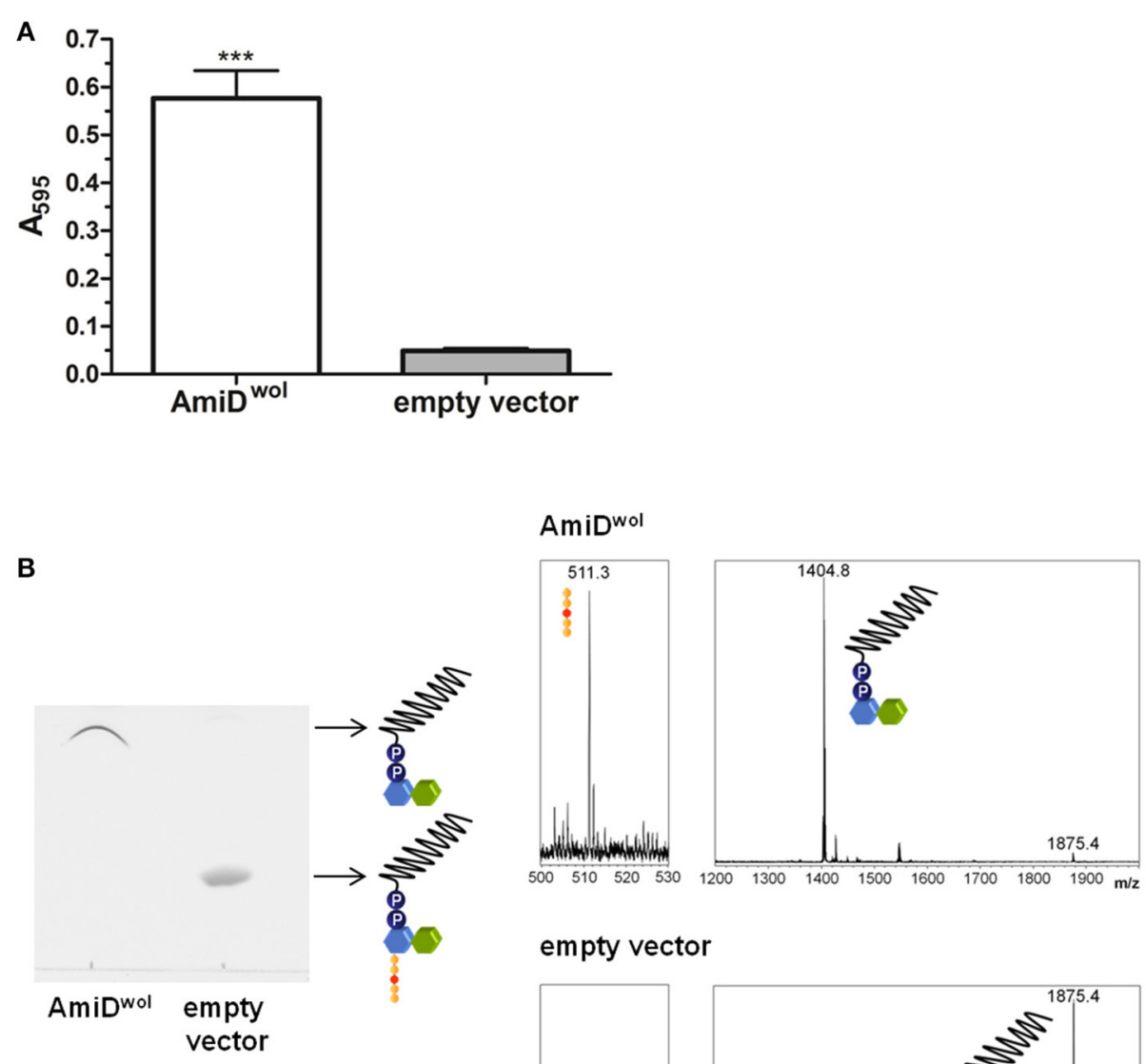

empty vector

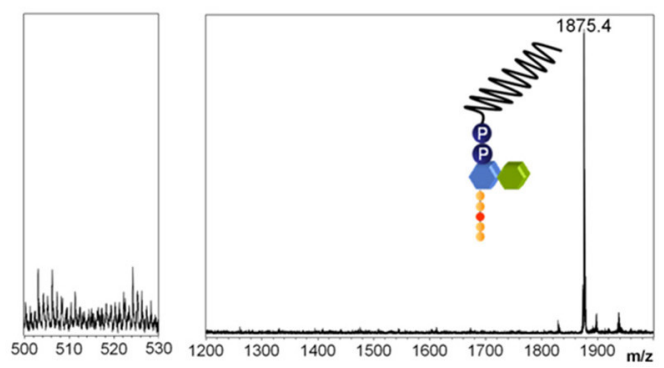

FIGURE 3 | AmiD ${ }^{\text {wol }}$ can use PG and lipid II as substrates in vitro. (A) Degradation of PG was detected by monitoring the absorbance at $595 \mathrm{~nm}$ of Remazol Brilliant Blue dye released into the supernatant after incubation with $\mathrm{AmiD}^{\text {wol }}$ overnight at $30^{\circ} \mathrm{C}$. Product from cells containing an empty expression vector was used as a negative control. Bars indicate mean \pm SEM. The graph is representative of six experiments with different batches of purified enzyme. (B). Lipid II was incubated with AmiD wol for $4 \mathrm{~h}$ at $30^{\circ} \mathrm{C}$. The reaction products were analyzed by TLC and MS (m/z-lipid II: 1,875.4; undecaprenyl-pyrophosphoryl-MurNAc-GlcNAc: 1,404.8; pentapeptide (sodium adduct): 511.3). Statistical difference was determined using student's $t$-test, with $P<0.05 .{ }^{* \star \star} P<0.001$.

Figure 2). Hence, all further activity tests were performed at $30^{\circ} \mathrm{C}$ and at $\mathrm{pH} 7.5$.

We next tested whether $\mathrm{AmiD}^{\text {wol }}$ can remove the pentapeptide from the cell wall precursor lipid II. Purified lipid II was incubated with $\mathrm{AmiD}^{\text {wol }}$ and the reaction products were extracted and analyzed by TLC and MS. In contrast to E. coli AmiD (Pennartz et al., 2009), AmiD ${ }^{\text {wol }}$ was able to use lipid II as a substrate and hydrolyzed the amide bond between MurNAc and L-Ala (Figure 3B).

E. coli AmiD has a broad substrate specificity and can also cleave anhydroMurNAc-L-Ala-bonds produced by lytic transglycosylases during cell growth (Uehara and Park, 2007). Therefore, TetraAnh and TetraTetradiAnh (Figure 4C) resulting from PG digested with the E. coli lytic transglycosylase Slt70 were incubated with $A$ miD ${ }^{\text {wol }}$. The products were separated by HPLC and confirmed by MS. AmiD ${ }^{\text {wol }}$ hydrolyzed TetraAnh and TetraTetradiAnh in a dose dependent manner (Figure 4A),

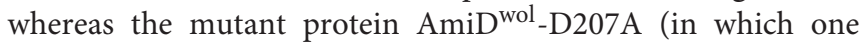
of the zinc-coordinating residues was replaced by alanine) was inactive (Figure 4B).

Together, these data demonstrate that $\mathrm{AmiD}^{\mathrm{wol}}$ is capable of cleaving intact PG, the cell wall precursor lipid II, as well as soluble PG fragments including the anhydro form.

\section{AmiD wol Activity Is Zinc-Dependent}

The active site of $\mathrm{AmiD}^{\text {wol }}$ is comprised of three conserved zinc-coordinating residues (H79, H187, D207; Figure 1 and Supplementary Figure 3 ) that are essential for amidase activity 

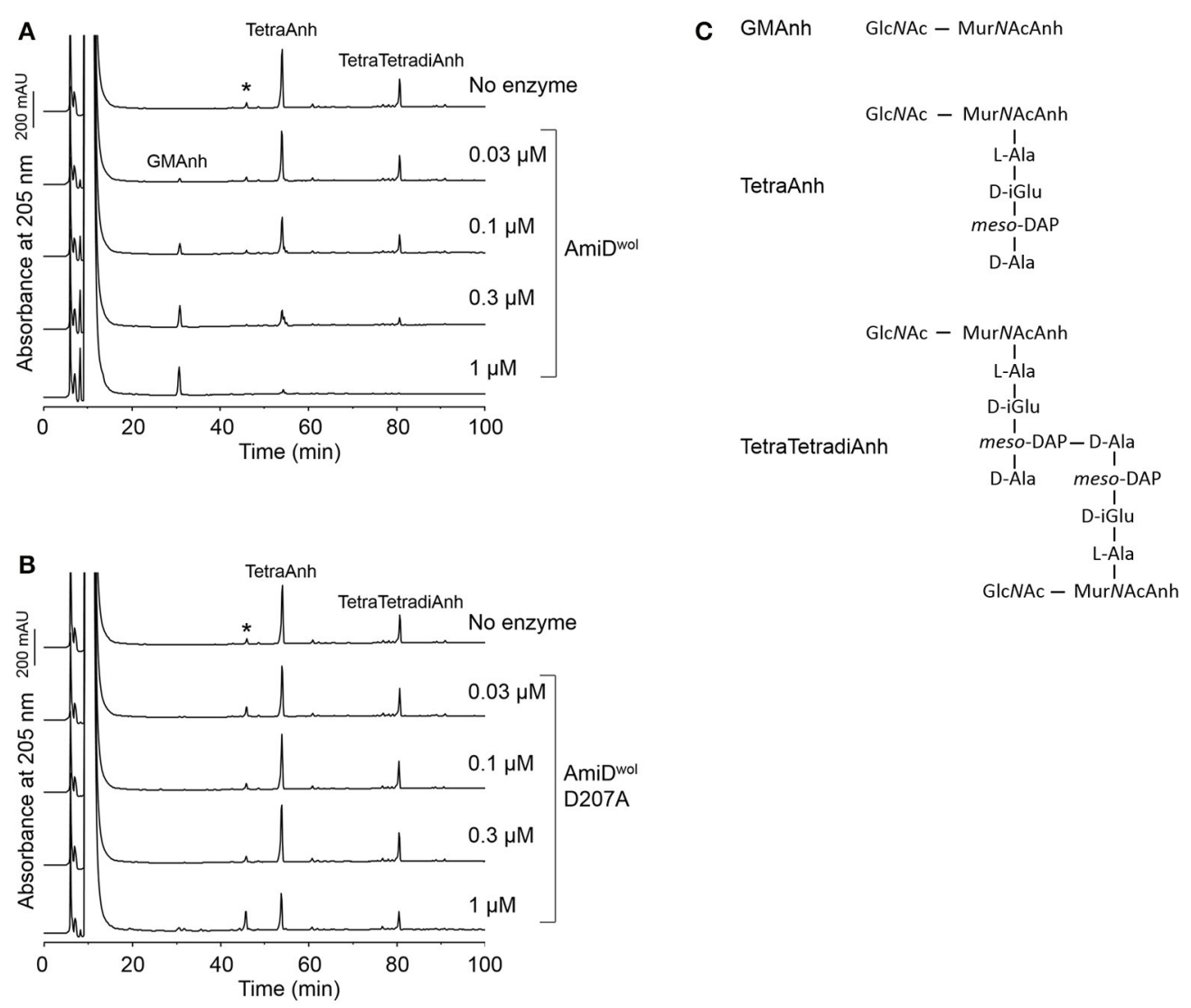

FIGURE 4 | AmiD ${ }^{\text {wol }}$ can use anhydromuropeptides as substrates. (A) Anhydromuropeptides (TetraAnh, TetraTetradiAnh) derived from PG digested with the E. coli Iytic transglyosylase SIt70 were incubated for $4 \mathrm{~h}$ at $30^{\circ} \mathrm{C}$ with $\mathrm{AmiD}^{\text {wol }}$ or (B) the amidase active-site mutant AmiD wol D207A at different concentrations. The samples were analyzed by HPLC and MS. The main product was identified as GlcNAc-MurNAcAnh ( $\mathrm{m} / \mathrm{z}=479.1866, \mathrm{H}^{+}$form; theoretical value: 479.1877).

(C) Structures of the muropeptides and the reaction products analyzed in $(\mathbf{A}, \mathbf{B})$. ${ }^{*}$ Unknown compound unrelated to the reaction.

(Uehara and Park, 2007; Kerff et al., 2010). PG and lipid II cleavage by $\mathrm{AmiD}^{\text {wol }}$ was decreased in the presence of EDTA and the specific $\mathrm{Zn}^{2+}$-chelator 1,10-phenanthroline (Figure 5A), although the enzyme activity was not completely inhibited by either compound, as has been shown for the E. coli ortholog (Uehara and Park, 2007). The mutation of one residue involved in zinc binding (H79A or D207A) resulted in a loss of activity, with $\mathrm{AmiD}^{\text {wol }}$-H79A and $\mathrm{AmiD}^{\text {wol }}$-D207A unable to hydrolyze the MurNAc-L-Ala bond in PG and lipid II (Figures 4B, 5B). Expression of the amidase active-site mutants $\mathrm{AmiD}^{\text {wol }}-\mathrm{H} 79 \mathrm{~A}$ and $\mathrm{AmiD}^{\text {wol }}$-D207A in E. coli also did not induce cell lysis in vivo (Figure 2).

\section{DISCUSSION}

Wolbachia are obligate intracellular bacteria with highly reduced genomes due to the adaption to- and dependency on their hosts (Moran and Plague, 2004). Interestingly, the genome of arthropod Wolbachia such as wMel encodes a peptidoglycan hydrolase (Wu et al., 2004), homologous to the MurNAc-L-Ala amidase AmiD, although a functional cell wall has not been detected.
In this study we characterized $\mathrm{AmiD}^{\text {wol }}$ to understand its role in Wolbachia biology. The presence of an N-terminal signal peptide predicts periplasmic localization of $\mathrm{AmiD}^{\mathrm{wol}}$. Our results demonstrate that the wolbachial periplasmic signal sequence can substitute for the OmpA leader peptide and that $\mathrm{AmiD}^{\mathrm{wol}}$ is indeed exported into the periplasm (Supplementary Figure 1). In contrast to the $E$. coli homolog, the $\mathrm{AmiD}^{\text {wol }}$ signal peptide does not include a cysteine-containing lipobox motif for further modification and insertion into the outer membrane (Figure 1). Thus, $\mathrm{AmiD}^{\text {wol }}$ is probably transported to the periplasm by the Sec pathway and cleaved between residues 30 and 31. The only Wolbachia lipoproteins identified from $w \mathrm{Bm}$ and insect Wolbachia pipientis strain A. albopictus $\mathrm{B}(w \mathrm{AlbB})$ to date are the peptidoglycan associated lipoprotein (wPAL) and the type IV secretion system component VirB6 (Voronin et al., 2014). Both are probably diacylated due to the absence of the gene encoding the $\mathrm{N}$-acetyltransferase Ltn.

This is the first description of a wolbachial lipid II processing enzyme putatively expressed in the periplasm. Our results demonstrate that $\mathrm{AmiD}^{\text {wol }}$ hydrolyzes the amide bond between MurNAc and L-Ala of various substrates as has been shown for E. coli AmiD. The functional conservation of $\mathrm{AmiD}^{\text {wol }}$ indicates 

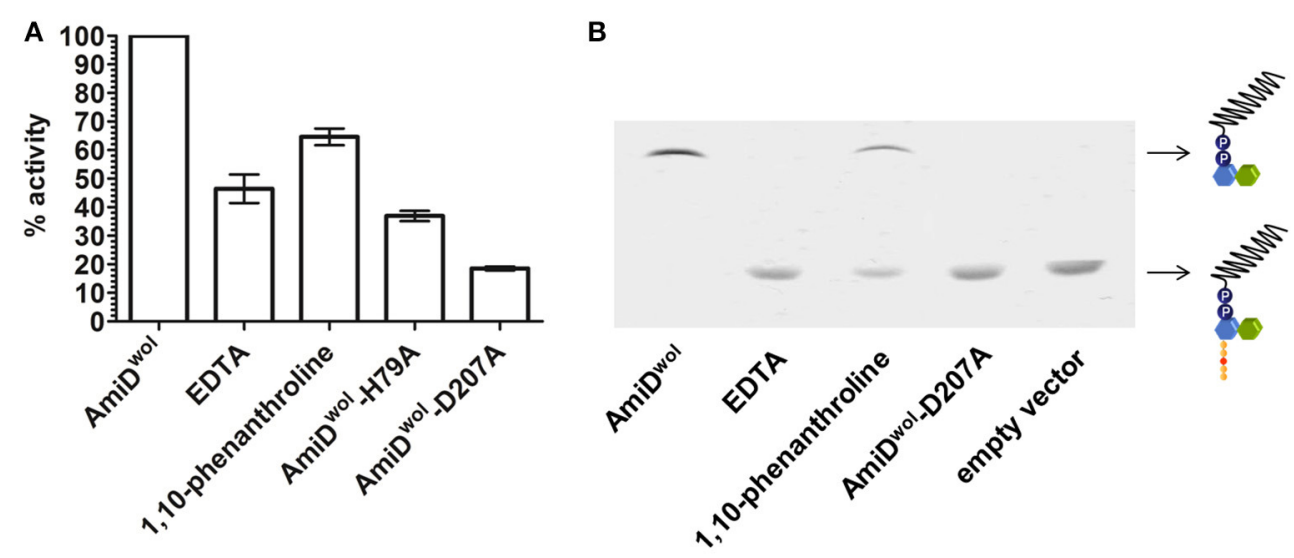

FIGURE 5 | Inhibition of AmiDwol activity. The zinc-dependent amidase activity could be inhibited in presence of 1 mM of the non-specific metal chelator EDTA and 5 $\mathrm{mM}$ of the $\mathrm{Zn}^{2+}$-specific chelator 1,10-phenanthroline as shown by (A) Remazol Brilliant Blue dye-release assay and (B) thin layer chromatography. Moreover, the exchange of one of the zinc-coordinating residues with alanine (H79A or D207A) decreased amidase activity. Results shown are means \pm SEM. The graph is representative of three experiments with different batches of purified enzyme.

that the ability to cleave anhydromuropeptides may play an important role in the lifestyle of insect Wolbachia and that these endobacteria may contain a PG-like structure with connected glycan strands because 1,6-anhydro bonds are generated by periplasmic lytic transglycosylases that cleave the glycosidic bond between MurNAc and GlcNAc units. However, neither a typical PG glycosyltransferase that could link the sugar moieties of the cell wall building block lipid II nor a lytic transglycosylase that could catalyze glycan chain cleavage during bacterial growth have been identified in the Wolbachia genomes (Wu et al., 2004; Foster et al., 2005). However, it has been recently suggested that RodA, a member of the SEDS (shape, elongation, division, sporulation) protein family, can polymerize glycan strands in the absence of known PG glycosyltransferases in Bacillus subtilis (Meeske et al., 2016). The absence of these enzymes in intracellular Chlamydia, Planctomycetes, and Wolbachia suggests that monofunctional PG transpeptidase-SEDS pairs might have been retained as principal PG polymerase systems (Henrichfreise et al., 2016). Supporting this hypothesis, recent studies demonstrate that PG can be detected and extracted from Chlamydia and Planctomycetes (Pilhofer et al., 2013; Liechti et al., 2014; Jeske et al., 2015; van Teeseling et al., 2015). It remains to be seen if the RodA/FtsW ortholog in Wolbachia has such an activity for the assembly of PG together with a monofunctional transpeptidase.

AmiA from Chlamydia pneumoniae has been demonstrated to rescue cell division in an E. coli strain deficient for AmiA, AmiB, and AmiC (Klöckner et al., 2014). E. coli AmiD was shown not to be involved in cell separation (Uehara and Park, 2007), therefore we would expect a similar result when using $\mathrm{AmiD}^{\text {wol }}$ expressed in E. coli deficient for the other amidases. Attempts were made to rescue such mutants, however expressed AmiD wol was half the expected size and no complementation was seen (data not shown). We cannot rule out that $\mathrm{AmiD}^{\mathrm{wol}}$ is involved in the turnover of a rudimentary PG-like structure as the recycling pathway of $\mathrm{C}_{55}-\mathrm{P}$ remains unclear (Henrichfreise et al., 2009; Vollmer et al., 2013). The exact role of $\mathrm{AmiD}^{\text {wol }}$ in lipid II/PG modification is still undefined. This raises the question why only AmiD is conserved in Wolbachia that behave more as parasites in arthropods.

In contrast to all other amidases in E. coli, AmiD has a broad substrate specificity and its role is still unclear. Uehara and Park (2007) proposed that the breakdown of cell wall fragments in the periplasm by AmiD is a secondary strategy to prevent immune responses in the host. Drosophila spp. relies entirely on innate immunity and two pathways respond to different classes of microorganisms (Buchon et al., 2014). The Toll pathway is mainly activated in response to Gram-positive bacteria and fungi, whereas the immune deficiency (Imd) pathway is mostly triggered by Gram-negative bacteria. Recognition of bacteria is mostly achieved by PG recognition proteins (PGRPs). The membrane anchored receptor PGRP-LC and the cytoplasmic receptor PGRP-LE upstream of the Imd pathway sense PG fragments containing $\mathrm{mDAP}$ in the peptide side chains found in Gram-negative bacteria and the Gram-positive bacilli (Buchon et al., 2014; Myllymaki et al., 2014). The minimum structure for recognition by PGRP-LC is a monomer of GlcNAc-MurNAc with an internal 1,6-anhydro-bond attached to a tripeptide (Stenbak et al., 2004). Thus, in addition to a possible role in processing lipid II for recycling, $\mathrm{AmiD}^{\text {wol }}$ might also help to suppress host immune responses by removing the peptide chain from the sugar moieties. Because insect Wolbachia are parasites and can horizontally infect other insects (Werren et al., 2008), this enzyme may have been maintained for example in $w \mathrm{Mel}$, Wolbachia from Drosophila simulans ( $w \mathrm{Ri})$ and Wolbachia from Culex quinquefasciatus Pel ( $w$ Pip) (Figure 1) to aid this specific endosymbiotic lifestyle and protect these endobacteria. In contrast, genomes of sequenced mutualistic Wolbachia from filarial nematodes (Foster et al., 2005) and the bedbug C. lectularis (Nikoh et al., 2014) show that these strains have lost the ability to synthesize $\mathrm{AmiD}^{\text {wol }}$. Moreover, nematodes do not express orthologs of PGRP-LC or Imd and thus would not recognize the same PG metabolism/recycling products, allowing Wolbachia 
of filarial nematodes to lose $A \mathrm{miD}^{\text {wol }}$ during evolution as mutualistic endosymbionts (Irazoqui et al., 2010; Ermolaeva and Schumacher, 2014). This hypothesis of AmiD as a protective mechanism will benefit from comparative analysis of Wolbachia genomes from all supergroups.

\section{AUTHOR CONTRIBUTIONS}

AH and KP conceptualized the study and obtained funding. $\mathrm{MW}, \mathrm{KM}, \mathrm{AS}, \mathrm{AK}, \mathrm{BH}$, and $\mathrm{KP}$ conceived and designed the experiments. MW, KM, MJ, $\mathrm{AK}$, and $\mathrm{CO}$ performed the laboratory experiments and analyzed data. MW, KM, and KP drafted the manuscript, with critical review by $\mathrm{AH}$ and WV.

\section{FUNDING}

The authors received support for this project from a German Research Foundation (Deutsche Forschungsgemeinschaft) grant to $\mathrm{KP}$ and $\mathrm{AH}$ (FOR 854, PF673/3-1 and PF673/3-2).

\section{REFERENCES}

Buchon, N., Silverman, N., and Cherry, S. (2014). Immunity in Drosophila melanogaster from microbial recognition to whole-organism physiology. Nat. Rev. Immunol. 14, 796-810. doi: 10.1038/nri3763

Bui, N. K., Gray, J., Schwarz, H., Schumann, P., Blanot, D., and Vollmer, W. (2009). The peptidoglycan sacculus of Myxococcus xanthus has unusual structural features and is degraded during glycerol-induced myxospore development. J. Bacteriol. 191, 494-505. doi: 10.1128/JB.00608-08

Ermolaeva, M. A., and Schumacher, B. (2014). Insight from the worm: the C. elegans model for innate immunity. Semin. Immunol. 26, 303-309. doi: 10.1016/j.smim.2014.04.005

Foster, J., Ganatra, M., Kamal, I., Ware, J., Makarova, K., Ivanova, N., et al. (2005). The Wolbachia genome of Brugia malayi: endosymbiont evolution within a human pathogenic nematode. PLoS Biol. 3:e121. doi: 10.1371/journal.pbio.0030121

Glauner, B. (1988). Separation and quantification of muropeptides with high-performance liquid-chromatography. Anal. Biochem. 172, 451-464. doi: 10.1016/0003-2697(88)90468-X

Heidrich, C., Templin, M. F., Ursinus, A., Merdanovic, M., Berger, J., Schwarz, $\mathrm{H}$., et al. (2001). Involvement of $\mathrm{N}$-acetylmuramyl-L-alanine amidases in cell separation and antibiotic-induced autolysis of Escherichia coli. Mol. Microbiol. 41, 167-178. doi: 10.1046/j.1365-2958.2001.02499.x

Henrichfreise, B., Brunke, M., and Viollier, P. H. (2016). Bacterial surfaces: the wall that SEDS built. Curr. Biol. 26, R1158-R1160. doi: 10.1016/j.cub.2016.09.028

Henrichfreise, B., Schiefer, A., Schneider, T., Nzukou, E., Poellinger, C., Hoffmann, T. J., et al. (2009). Functional conservation of the lipid II biosynthesis pathway in the cell wall-less Chlamydia and Wolbachia: why is lipid II needed? Mol. Microbiol. 73, 913-923. doi: 10.1111/j.1365-2958.2009.06815.x

Irazoqui, J. E., Urbach, J. M., and Ausubel, F. M. (2010). Evolution of host innate defense: insights from Caenorhabditis elegans and primitive invertebrates. Nat. Rev. Immunol. 10, 47-58. doi: 10.1038/nri2689

Jacobs, C., Joris, B., Jamin, M., Klarsov, K., Van Beeumen, J., Mengin-Lecreulx, D., et al. (1995). AmpD, essential for both beta-lactamase regulation and cell wall recycling, is a novel cytosolic N-acetylmuramyl-L-alanine amidase. Mol. Microbiol. 15, 553-559. doi: 10.1111/j.1365-2958.1995.tb02268.x

Jeske, O., Schüler, M., Schumann, P., Schneider, A., Boedeker, C., Jogler, M., et al. (2015). Planctomycetes do possess a peptidoglycan cell wall. Nat. Commun. 6:7116. doi: $10.1038 /$ ncomms 8116

Johnson, J. W., Fisher, J. F., and Mobashery, S. (2013). Bacterial cell-wall recycling. Ann. N.Y. Acad. Sci. 1277, 54-75. doi: 10.1111/j.1749-6632.2012.06813.x
$\mathrm{AH}$ is a member of the German Center for Infection Research (DZIF). $\mathrm{AH}$ is a member and $\mathrm{BH}$ and $\mathrm{KP}$ are associate members of the Excellence Cluster Immunosensation (DFG, EXC 1023). BH received support by the intramural funding scheme of the Medical Faculty of Bonn, BONFOR. Work in WV lab was supported by the UK Medical Research Council within the JPI AMR project NAPCLI.

\section{ACKNOWLEDGMENTS}

We thank Joe Gray (Pinnacle laboratory, Newcastle University) for mass spectrometry analysis of muropeptides.

\section{SUPPLEMENTARY MATERIAL}

The Supplementary Material for this article can be found online at: http://journal.frontiersin.org/article/10.3389/fcimb. 2017.00353/full\#supplementary-material

Kerff, F., Petrella, S., Mercier, F., Sauvage, E., Herman, R., Pennartz, A., et al. (2010). Specific structural features of the $\mathrm{N}$-acetylmuramoyl-L-alanine amidase AmiD from Escherichia coli and mechanistic implications for enzymes of this family. J. Mol. Biol. 397, 249-259. doi: 10.1016/j.jmb.2009.12.038

Klöckner, A., Otten, C., Derouaux, A., Vollmer, W., Bühl, H., De Benedetti, S., et al. (2014). AmiA is a penicillin target enzyme with dual activity in the intracellular pathogen Chlamydia pneumoniae. Nat. Commun. 5:4201. doi: $10.1038 /$ ncomms5201

Lenz, J. D., Stohl, E. A., Robertson, R. M., Hackett, K. T., Fisher, K., Xiong, K., et al. (2016). Amidase activity of AmiC controls cell separation and stem peptide release and is enhanced by NlpD in Neisseria gonorrhoeae. J. Biol. Chem. 291, 10916-10933. doi: 10.1074/jbc.M116.715573

Liechti, G. W., Kuru, E., Hall, E., Kalinda, A., Brun, Y. V., VanNieuwenhze, M., et al. (2014). A new metabolic cell-wall labelling method reveals peptidoglycan in Chlamydia trachomatis. Nature 506, 507-510. doi: 10.1038/nature12892

Lindsey, A. R., Werren, J. H., Richards, S., and Stouthamer, R. (2016). Comparative genomics of a parthenogenesis-inducing Wolbachia symbiont. G3 6, 2113-2123. doi: 10.1534/g3.116.028449

Meeske, A. J., Riley, E. P., Robins, W. P., Uehara, T., Mekalanos, J. J., Kahne, D., et al. (2016). SEDS proteins are a widespread family of bacterial cell wall polymerases. Nature 537, 634-638. doi: 10.1038/nature19331

Möll, A., Dörr, T., Alvarez, L., Chao, M. C., Davis, B. M., Cava, F., et al. (2014). Cell separation in Vibrio cholera is mediated by a single amidase whose action is modulated by two nonredundant activators. J. Bacteriol. 196, 3937-3948. doi: 10.1128/JB.02094-14

Moran, N. A., and Plague, G. R. (2004). Genomic changes following host restriction in bacteria. Curr. Opin. Genet. Dev. 14, 627-633. doi: 10.1016/j.gde.2004.09.003

Myllymaki, H., Valanne, S., and Ramet, M. (2014). The Drosophila imd signaling pathway. J. Immunol. 192, 3455-3462. doi: 10.4049/jimmunol.1303309

Nikoh, N., Hosokawa, T., Moriyama, M., Oshima, K., Hattori, M., and Fukatso, T. (2014). Evolutionary origin of insect-Wolbachia nutritional mutualism. Proc. Natl. Acad. Sci. U.S.A. 111, 10257-10262. doi: 10.1073/pnas.1409284111

Otten, C., De Benedetti, S., Gaballah, A., Bühl, H., Klöckner, A., Brauner, J., et al. (2015). Co-solvents as stabilizing agents during heterologous overexpression in Escherichia coli - application to chlamydial penicillin-binding protein 6. PLoS ONE 10:e0122110. doi: 10.1371/journal.pone.0122110

Pennartz, A., Genereux, C., Parquet, C., Mengin-Lecreulx, D., and Joris, B. (2009). Substrate-induced inactivation of the Escherichia coli AmiD NAcetylmuramoyl-L-Alanine amidase highlights a new strategy to inhibit this class of enzyme. Antimicrob. Agents Chemother. 53, 2991-2997. doi: 10.1128/AAC.01520-07 
Pilhofer, M., Aistleitner, K., Biboy, J., Gray, J., Kuru, E., Hall, E., et al. (2013). Discovery of chlamydial peptidoglycan reveals bacteria with murein sacculi but without FtsZ. Nat. Commun. 4:2856. doi: 10.1038/ncomms3856

Priyadarshini, R., de Pedro, M. A., and Young, K. D. (2007). Role of peptidoglycan amidases in the development and morphology of the division septum in Escherichia coli. J. Bacteriol. 189, 5334-5347. doi: 10.1128/JB.00415-07

Rouser, G., Fleischer, S., and Yamamoto, A. (1970). Two dimensional then layer chromatographic separation of polar lipids and determination of phospholipids by phosphorus analysis of spots. Lipids 5, 494-496. doi: 10.1007/BF02531316

Schneider, T., Senn, M. M., Berger-Bachi, B., Tossi, A., Sahl, H. G., and Wiedemann, I. (2004). In vitro assembly of a complete, pentaglycine interpeptide bridge containing cell wall precursor (lipid II-Gly5) of Staphylococcus aureus. Mol. Microbiol. 53, 675-685. doi: 10.1111/j.1365-2958.2004.04149.x

Specht, S., Debrah, A. Y., Klarmann, U., Mand, S., Hoerauf, A., and Pfarr, K. (2013). Chemotherapy of filariasis - established strategies and new developments. GMS Infect. Dis. 1, 1-10. doi: 10.3205/id000003

Stenbak, C. R., Ryu, J. H., Leulier, F., Pili-Floury, S., Parquet, C., Herve, M., et al. (2004). Peptidoglycan molecular requirements allowing detection by the Drosophila immune deficiency pathway. J. Immunol. 173, 7339-7348. doi: 10.4049/jimmunol.173.12.7339

Stepkowski, T., and Legocki, A. B. (2001). Reduction of bacterial genome size and expansion resulting from obligate intracellular lifestyle and adaptation to soil habitat. Acta Biochim. Pol. 48, 367-381.

Taylor, M. J., and Hoerauf, A. (2001). A new approach to the treatment of filariasis. Curr. Opin. Infect. Dis. 14, 727-731. doi: 10.1097/00001432-200112000-00011

Taylor, M. J., Hoerauf, A., and Bockarie, M. (2010). Lymphatic filariasis and onchocerciasis. Lancet 376, 1175-1185. doi: 10.1016/S0140-6736(10)60586-7

Typas, A., Banzhaf, M., Gross, C. A., and Vollmer, W. (2012). From the regulation of peptidoglycan synthesis to bacterial growth and morphology. Nat. Rev. Microbiol. 10, 123-136. doi: 10.1038/nrmicro2677

Uehara, T., Dinh, T., and Bernhardt, T. G. (2009). LytM-domain factors are required for daughter cell separation and rapid ampicillin-induced lysis in Escherichia coli. J. Bacteriol. 191, 5094-5107. doi: 10.1128/JB.00505-09

Uehara, T., and Park, J. T. (2007). An anhydro-N-acetylmuramyl-L-alanine amidase with broad specificity tethered to the outer membrane of Escherichia coli. J. Bacteriol. 189, 5634-5641. doi: 10.1128/JB.00446-07

Uehara, T., Parzych, K. R., Dinh, T., and Bernhardt, T. G. (2010). Daughter cell separation is controlled by cytokinetic ring-activated cell wall hydrolysis. EMBO J. 29, 1412-1422. doi: 10.1038/emboj.2010.36 van Teeseling, M. C., Mesman, R. J., Kuru, E., Espaillat, A., Cava, F., Brun, Y. V., et al. (2015). Annamox planctomycetes have a peptidoglycan cell wall. Nat. Commun. 6:6878. doi: 10.1038/ncomm s7878

Vollmer, J., Schiefer, A., Schneider, T., Jülicher, K., Johnston, K. L., Taylor, M. J., et al. (2013). Requirement of lipid II biosynthesis for cell division in cell wall-less Wolbachia, endobacteria of arthropods and filarial nematodes. Int. J. Med. Microbiol. 303, 140-149. doi: 10.1016/j.ijmm.2013. 01.002

Vollmer, W., Joris, B., Charlier, P., and Foster, S. (2008). Bacterial peptidoglycan (murein) hydrolases. FEMS Microbiol. Rev. 32, 259-286. doi: 10.1111/j.1574-6976.2007.00099.x

Voronin, D., Guimaraes, A. F., Molyneux, G. R., Johnston, K. L., Ford, L., and Taylor, M. J. (2014). Wolbachia lipoproteins: abundance, localisation and serology of Wolbachia peptidoglycan associated lipoprotein and the type IV secretion system component, VirB6 from Brugia malayi and Aedes albopictus. Parasit. Vectors 7:462. doi: 10.1186/s13071-0140462-1

Werren, J. H., Baldo, L., and Clark, M. E. (2008). Wolbachia: master manipulators of invertebrate biology. Nat. Rev. Microbiol. 6, 741-751. doi: 10.1038/nrmicro1969

Wu, M., Sun, L. V., Vamathevan, J., Riegler, M., Deboy, R., Brownlie, J. C., et al. (2004). Phylogenomics of the reproductive parasite Wolbachia pipientis w Mel: a streamlined genome overrun by mobile genetic elements. PLoS Biol. 2:E69. doi: 10.1371/journal.pbio.0020069

Zug, R., and Hammerstein, P. (2015). Bad guys turned nice? A critical assessment of Wolbachia mutualism in arthropod hosts. Biol. Rev. Camb. Philos. Soc. 90, 89-111. doi: 10.1111/brv.12098

Conflict of Interest Statement: The authors declare that the research was conducted in the absence of any commercial or financial relationships that could be construed as a potential conflict of interest.

Copyright (C) 2017 Wilmes, Meier, Schiefer, Josten, Otten, Klöckner, Henrichfreise, Vollmer, Hoerauf and Pfarr. This is an open-access article distributed under the terms of the Creative Commons Attribution License (CC BY). The use, distribution or reproduction in other forums is permitted, provided the original author(s) or licensor are credited and that the original publication in this journal is cited, in accordance with accepted academic practice. No use, distribution or reproduction is permitted which does not comply with these terms. 